\title{
Les impacts psychologiques des métiers dangereux : Portrait de situation de la santé psychologique des pompiers permanents québécois
}

\author{
Jacinthe Douesnard, Ph. D. \\ Université du Québec à Chicoutimi
}

\section{INTRODUCTION}

\section{Le travail est un lieu de réalisations personnelles pour une grande majorité d'individus, mais peut également engendrer des problèmes de santé psychologique chez les travailleurs.}

Le travail est un lieu de réalisations personnelles pour une grande majorité d'individus ${ }^{1}$, mais peut également engendrer des problèmes de santé psychologique chez les travailleurs. En effet, plusieurs études effectuées dans différents milieux de travail permettent de bien documenter les facteurs de risque présents dans le travail et leurs impacts sur la santé psychologique des travailleurs ${ }^{2}$. Ces constats mènent à s'interroger

\section{MISE EN CONTEXTE}

\subsection{Facteurs de risque inhérents au métier de pompier}

\section{Dans bien des interventions, les pompiers sont exposés à des situations dangereuses, menaçant leur propre vie, celle de leurs collègues ou celle des citoyens.}

Dans les milieux incendies, il est possible de retrouver des situations éprouvantes et exigeantes tant sur le plan physique que mental. Ces exigences, inhérentes au métier de pompier, représentent des facteurs de risque d'atteinte à leur santé psychologique. Par exemple, dans bien des interventions, les pompiers sont exposés à des situations dangereuses, menaçant leur propre vie, celle de leurs collègues ou celle des citoyens. Ils peuvent également être témoins de pertes matérielles importantes, de souffrances humaines, de blessures graves, de brûlures et plus particulièrement au sujet de la situation d'un groupe de travailleurs précis, soit les pompiers, lesquels œuvrent dans un environnement comportant de nombreux risques d'atteinte à leur santé psychologique. Étonnamment, malgré l'ampleur des risques auxquels ils sont exposés quotidiennement, très peu d'études abordent la question de la santé psychologique des pompiers, du moins, au Québec.

\section{Malgré l'ampleur des risques auxquels ils sont exposés quotidiennement, très peu d'études abordent la question de la santé psychologique des pompiers, du moins, au Québec.}

même de la mort. En tant qu'intervenants d'urgence, ils sauvent des victimes, retirent des personnes mortes des décombres, assistent à des scènes éprouvantes émotionnellement ${ }^{3}$ et ils ont la plupart du temps à intervenir rapidement et à poser des actions lourdes de conséquences. Lors d'une catastrophe, les demandes les plus pressantes reposent sur les épaules des répondants de première ligne (pompiers, policiers, employés des services médicaux et ambulanciers) ${ }^{4}$. Le travail de pompier est demandant, complexe, chargé de responsabilités et le rythme de travail est particulier. En effet, certains auteurs évoquent par exemple les temps de travail inégaux des intervenants en situation d'urgence, c'est-àdire l'absence (ou les trop brèves périodes) de repos entre les interventions, jumelés avec le travail échelonné sur de longues heures ${ }^{5}$. Les pompiers sont susceptibles d'être présents sur les lieux de l'intervention pendant une période im- 
portante, dépassant parfois le temps normal de travail. Ils peuvent également être appelés à ressortir de la caserne dès qu'ils rentrent d'un appel, n'ayant parfois pas le temps de reprendre l'énergie nécessaire pour être efficaces lors de l'intervention suivante. À l'opposé, les pompiers peuvent être pendant de longues heures en souscharge lors d'un quart de travail. Par conséquent, ils sont susceptibles de vivre de l'ennui lors de ces périodes, en attente d'un appel. Il est alors possible de constater que les exigences physiques et psychologiques liées à ce métier sont imprévisibles et ne sont pas constantes.

Considérant la description de leur métier, les exigences qui y sont liées ainsi que les facteurs de risque auxquels les pompiers sont exposés, l'Organisation internationale du travail ${ }^{6}$ affirme que le stress est inhérent à ce métier et que les exigences relatives à la nature du travail font des pompiers une population de travailleurs qui peut être exposée à des incidents critiques et développer des répercussions psychologiques négatives.

\subsection{La santé psychologique des pompiers dans la littérature scientifique}

En dépit des risques élevés présents dans le métier de pompier, peu d'études font état des conséquences psychologiques liées à ce travail.

En dépit des risques élevés présents dans le métier de pompier, peu d'études font état des conséquences psychologiques liées à ce travail. D'ailleurs, il est récent dans la littérature scientifique que des auteurs s'intéressent aux conséquences psychologiques que vivent les intervenants en situation d'urgence ${ }^{3}$. De fait, ce n'est que depuis la fin des années 1980 que les services incendie ont commencé à porter une attention aux impacts du travail des pompiers sur leur santé psychologique?

À travers le monde, les études relatives à la santé psychologique des pompiers portent en grande majorité sur le stress post-traumatique ${ }^{8}$ ou abordent la problématique selon l'approche cognitive et mettent alors l'accent sur l'effet des stratégies d'adaptation utilisées par les pompiers ${ }^{9}$ ou en- core du soutien social ${ }^{10}$. Par ailleurs, la plupart des recherches qui concernent les pompiers traitent habituellement d'un groupe bien précis de pompiers, soit ceux qui ont assisté à un événement d'ampleur, tel un écrasement d'avion ${ }^{11}$ ou encore des désastres naturels ${ }^{12}$.

Bien qu'il existe des études portant sur l'état de santé psychologique des pompiers dans certains pays (Japon, Allemagne, Australie, Irlande, États-Unis, Canada), et bien que ces recherches soient pertinentes, nous constatons tout de même un vide dans le portrait de la littérature scientifique au sujet de la santé psychologique des pompiers québécois. De plus, il faut être prudent dans l'interprétation des données contenues dans les recherches provenant d'autres pays, puisque ces données peuvent difficilement être transposables au Québec, étant donné le caractère unique de chaque organisation de service incendie. À titre d'exemple, les sapeurs-pompiers, en France, sont pour la plupart des militaires. La formation est par conséquent très différente de celle que reçoivent les pompiers québécois et l'organisation du travail diffère également.

Cet article propose donc de décrire la prévalence des problèmes de santé psychologique chez les pompiers québécois. Pour ce faire, nous présentons tout d'abord la méthodologie qui a été utilisée pour cette recherche. Puis, sont présentés les résultats portant notamment sur la prévalence de certains indicateurs de santé psychologiques, soit la détresse psychologique, l'épuisement professionnel et le stress post-traumatique. Ces prévalences sont comparées à d'autres prévalences obtenues auprès de la population canadienne et québécoise de travailleurs ou auprès de population de travailleurs qui œuvrent dans des milieux de travail similaire. Enfin, cet article présente une discussion au sujet des prévalences et des questionnements qui en découlent.

À travers le monde, les études relatives à la santé psychologique des pompiers portent en grande majorité sur le stress post-traumatique ou abordent la problématique selon l'approche cognitive et mettent alors l'accent sur l'effet des stratégies d'adaptation utilisées par les pompiers ou encore du soutien social. 


\section{MÉTHODOLOGIE}

\subsection{Population à l'étude}

La population à l'étude est composée de pompiers permanents travaillant dans deux des six services incendie de la province de Québec employant majoritairement des pompiers à temps plein (pompiers permanents). La présente étude s'est déroulée auprès de pompiers permanents afin d'avoir une population de travailleurs qui ont le métier de pompier comme principale occupation professionnelle, en vivant toutes les dimensions que cela implique, c'est-à-dire les interventions ainsi que le temps passé en caserne entre collègues à attendre les alarmes.

\subsection{Procédures}

Cette étude s'est déroulée selon un devis de recherche quantitatif, à l'aide d'un questionnaire qui a été distribué en mains propres à tous les pompiers qui étaient présents lors de notre passage en caserne afin d'obtenir des résultats appuyés sur un nombre de réponses le plus important possible et d'ainsi assurer la représentativité des résultats. De fait, le questionnaire a été distribué dans 25 casernes. Étant donné que chaque caserne compte quatre (4) quarts de travail, il y a donc eu 100 groupes de travail qui ont reçu le questionnaire. Rencontrer chaque groupe de travail des deux villes et être présente sur place pour remettre le questionnaire en mains propres et le récupérer a permis de bien expliquer le projet aux participants et de répondre à leurs interrogations. À la suite de l'explication du projet, le questionnaire était remis à ceux qui acceptaient de participer à l'étude, puisque la participation se faisait sur une base volontaire.

\subsection{Outil : le questionnaire et ses variables}

Le questionnaire permettait, entre autres, de mesurer trois principaux troubles psychologiques : la détresse psychologique, l'épuisement professionnel ainsi que le stress post-traumatique.

Le questionnaire, composé de questions fermées, couvre certaines dimensions de la santé psychologique. Il a été élaboré en combinant diverses échelles existantes et déjà validées, rendant possible une comparaison avec d'autres données recueillies dans des recherches similaires. Plus précisément, le questionnaire permettait, entre autres, de mesurer trois principaux troubles psychologiques: la détresse psychologique, l'épuisement professionnel ainsi que le stress post-traumatique.

Premièrement, 1'échelle K- $6^{13}$ permet de mesurer la détresse psychologique. Cet outil, utilisé notamment dans l'Enquête sur la santé dans les collectivités canadiennes (ESCC) et dans l'Étude québécoise des conditions de travail, d'emploi et de santé et sécurité du travail (EQCOTESST) ${ }^{14}$, comporte six (6) questions. Ces questions mesurent la fréquence, dans le dernier mois, à laquelle le travailleur s'est senti nerveux, désespéré, agité, déprimé, bon à rien et qu'il a eu l'impression que tout était un effort. Le K-6 est un sous-ensemble de questions du Composite International Diagnostic Interview (CIDI) de l'Organisation mondiale de la santé. Les qualités psychométriques de l'outil ont été démontrées ${ }^{15}$.

Deuxièmement, l'épuisement professionnel est mesuré à l'aide du Copenhagen Burnout Inventory $(\mathrm{CBI})^{16}$. Une des trois sous-échelles du CBI, composée de sept (7) items, est utilisée afin d'identifier le degré d'épuisement psychologique du travailleur relativement à son métier (frustrations associées au travail, épuisement émotionnel lié au travail, etc.).

Troisièmement, le trouble de stress post-traumatique (TSPT) est mesuré par l'échelle EMST (Modified PTSD Symptoms Scale) ${ }^{17}$. Cette échelle de 17 items mesure la fréquence et la sévérité des symptômes du TSPT tels que ressentis par l'individu au cours des deux dernières semaines. Le participant qui répond par l'affirmative à une question filtre introduite dans le questionnaire, laquelle identifie si la personne a été confrontée à un événement traumatisant, doit identifier par la suite la sévérité (de « pas du tout perturbant » à 《extrêmement perturbant ») et la fréquence (de «jamais » à «presque toujours ») de 17 symptômes caractérisant le trou- 
ble de stress post-traumatique. Les questions portent entre autres sur des souvenirs intrusifs, des mauvais rêves liés à l'événement, la perte d'intérêt dans les activités quotidiennes, une perturbation dans les émotions, etc. Cette échelle a été validée ${ }^{18}$ dans un milieu francophone, auprès d'un échantillon clinique québécois.

Finalement, une question a été ajoutée afin de mesurer la perception qu'ont les pompiers de certains stresseurs présents dans leur métier. Ils étaient invités à attribuer une note (de 1 à 5) à chacun des items qui leur étaient présentés afin de quantifier l'impact que peuvent avoir ces éléments sur leur santé psychologique, autrement dit, sur la façon dont ils se sentent. Les 28 items présentés dans le questionnaire ont été tirés d'une recherche faite auprès des sapeurspompiers en France ${ }^{19}$. Dans cette recherche étaient énumérés tous les éléments nommés par les pompiers français comme étant dérangeants dans leur travail. Cette liste d'items a été reprise et présentée aux pompiers québécois dans le cadre de la recherche afin qu'ils déterminent, pour chaque élément, s'il s'agit d'une facette de leur travail qui a un impact «très peu négatif » (1) ou « extrêmement négatif » (5) sur leur santé psychologique. Par exemple, est-ce que le fait d'avoir à intervenir dans une situation impliquant un enfant est un élément de leur travail qui a un impact négatif sur eux (très peu à extrêmement).

\subsection{Méthode d'analyse des données du questionnaire}

Des mesures de fréquence ont été effectuées afin de tracer le portrait de la situation et de mesurer la prévalence de certains facteurs relatifs à la santé psychologique. Les prévalences présentées dans la section « résultats » sont brutes, c'est-àdire qu'aucun ajustement pour l'âge, la ville, l'ancienneté ou le poste n'a été effectué puisque ces ajustements ne changeaient pas les prévalences brutes de façon significative.

La détresse psychologique est mesurée en faisant une sommation des items composant l'échelle K-6. Le score d'un répondant peut se situer entre 0 et 24. Plus le score est élevé, plus la détresse psychologique est élevée. Nous présentons la prévalence de la détresse psychologique calculée selon deux méthodes (deux seuils).

D'abord, afin de permettre une comparaison avec la prévalence obtenue dans l'Enquête sur la santé dans les collectivités canadiennes menée par Santé Canada, la valeur seuil de 5 a été utilisée pour déterminer les sujets exposés ou non exposés à de la détresse psychologique ${ }^{20}$. La valeur seuil signifie que les sujets exposés à de la détresse psychologique sont ceux ayant un score égal ou supérieur à 5 .

Ensuite, nous présentons également la prévalence obtenue lorsque la valeur seuil de 7 est utilisée, tel que les chercheurs de l'Étude québécoise des conditions de travail, d'emploi et de santé et sécurité du travail ${ }^{14}$ le proposent. En ce sens, les sujets ayant obtenu un score égal ou supérieur à 7 sont considérés comme présentant les signes liés à de la détresse psychologique.

Finalement, les scores de la détresse psychologique sont également classés par catégories, selon la gravité des symptômes associés à chaque score. Pour ce faire, nous utilisons les catégories présentées dans l'Étude québécoise des conditions de travail, d'emploi et de santé et sécurité $\mathrm{du}$ travail $^{14}$. Ainsi, un score de 0 signifie qu'aucune détresse psychologique n'est présente, un score qui se situe entre 1 et 4 signifie de la détresse légère, entre 5-6 pour la détresse modérée, entre 7 et 12 pour la détresse élevée et un score de 13 et plus signifie de la détresse sévère.

Concernant l'épuisement professionnel, un score total est donné à chaque répondant. Ces scores ont été obtenus par la sommation des items qui composaient le CBI. Les répondants ayant obtenu un score supérieur à 50 sont considérés comme présentant de l'épuisement professionnel, tel que recommandé par les auteurs de l'échelle. Les prévalences obtenues par les pompiers sont comparées à celles obtenues par les préposés des centrales 9-1-1 au Québec (ci-après appelés préposés 9-1-1) ${ }^{21}$. Cette population de travailleur a été utilisée comme référence de comparaison puisque les mêmes outils et les mêmes méthodes d'analyses ont été utilisés auprès de ces travailleurs. 
Finalement, le trouble de stress post-traumatique (TSPT) est évalué à l'aide de deux sous-mesures, soit la sévérité des symptômes et la fréquence des symptômes, tel que proposé par les auteurs de l'échelle du TSPT. Pour la première sous-mesure (sévérité), les répondants qui obtiennent un score supérieur à 27 sont considérés comme ayant des symptômes sévères de TSPT et ceux obtenant un score supérieur à 21 à la sous-mesure de fréquence, sont considérés comme ayant fréquemment des symptômes liés au TSPT. La prévalence du stress post-

\section{RÉSULTATS}

\subsection{Taux de réponse}

Sur les 584 travailleurs que comptaient au total les deux services incendie au moment de la passation des questionnaires, 429 pompiers ont été rencontrés. Sur cette portion de 429 pompiers qui ont été rencontrés, 423 ont accepté de répondre au questionnaire, ce qui représente un taux de réponse de 98,6\%. De façon plus globale, 423 pompiers sur les 584 pompiers que comptent au total les deux services ont participé à la recherche, ce qui signifie que $72 \%$ du nombre total des pompiers ont répondu au questionnaire.

\subsection{Prévalences de la détresse psychologique}

En considérant les scores totaux supérieurs à 5 (valeur seuil de l'Enquête sur la santé dans les traumatique en général est obtenue en déterminant ceux dont le score total se situe audelà de 50. Une autre méthode pour obtenir la prévalence du TSPT en général est de considérer ceux qui sont exposés aux deux sous-mesures (sévérité et fréquence) comme étant atteints de stress post-traumatique. Les résultats de ces deux types de mesures générales sont présentés. Ces prévalences sont également comparées à celles des préposés 9-1-1, comme il a été le cas pour l'épuisement professionnel. collectivités canadiennes), on obtient pour les pompiers une prévalence de $28 \%$ quant à la détresse psychologique (tableau 1). Ce résultat peut être comparé à celui que l'on retrouve dans l'Enquête sur la santé dans les collectivités canadiennes indiquant que $24 \%$ des travailleurs canadiens ont connu un épisode de détresse psychologique au cours des quatre années visées par l'enquête canadienne (entre 1994-1995 et 2000$2001)^{19}$. De l'Enquête sur la santé dans les collectivités canadiennes effectuée auprès de la population canadienne, les données touchant plus précisément le Québec ont été analysées ${ }^{22}$ et la prévalence de détresse psychologique propre aux travailleurs québécois (aussi calculée en utilisant 5 comme valeur seuil) est de $25 \%$.

Tableau 1 : Comparaison des prévalences obtenues avec le K6 et une valeur seuil de 5 pour la détresse psychologique entre les pompiers, les travailleurs canadiens et les travailleurs québécois

\begin{tabular}{l|c}
\hline \multicolumn{1}{c|}{ POPULATION DE COMPARAISON } & $\begin{array}{c}\text { Prévalences } \\
\text { (obtenue avec la valeur seuil de 5) }\end{array}$ \\
\hline Pompiers & $\mathbf{2 8 ~ \%}$ \\
Population des travailleurs canadiens (ESCC) & $24 \%$ \\
Population des travailleurs québécois (ESCC) & $25 \%$ \\
\hline
\end{tabular}

D'autre part, utilisant le score 7 comme valeur seuil, tel qu'utilisé dans l'Étude québécoise des conditions de travail, d'emploi et de santé et sécurité du travail ${ }^{20}$, la prévalence des pompiers en détresse psychologique est de $14 \%$ (tableau
2). Ce résultat est plus faible que celui obtenu auprès de la population des travailleurs québécois dans l'Étude québécoise des conditions de travail, d'emploi et de santé et sécurité du travail, qui est de $19 \%$. 
Tableau 2 : Comparaison des prévalences obtenues avec le K6 et une valeur seuil de 7 pour la détresse psychologique entre les pompiers et les travailleurs québécois

\begin{tabular}{l|c}
\hline \multicolumn{1}{c|}{ PoPUlation DE COMPARAISON } & $\begin{array}{c}\text { Prévalences } \\
\text { (obtenue avec la valeur seuil de 7) }\end{array}$ \\
\hline Pompiers & $\mathbf{1 4 \%}$ \\
Travailleurs québécois (EQCOTESST) & $19 \%$ \\
\hline
\end{tabular}

Finalement, le tableau 3 présente en détail les catégories permettant de classer les différents scores obtenus à l'échelle K6, selon la gravité des symptômes. Ainsi, il est possible de constater que $6 \%$ des pompiers ne présentent aucun signe de détresse psychologique alors que la majorité $(66 \%)$ d'entre eux n'ont qu'une dé- tresse psychologique faible. Puis, $14 \%$ des répondants montrent des symptômes de détresse psychologique modérée, alors qu'un peu moins $(13 \%)$ d'entre eux obtiennent un score qui réfère à une détresse psychologique élevée. Finalement, seulement $1 \%$ des répondants sont en détresse psychologique très élevée.

Tableau 3 : Comparaison des prévalences obtenues avec le K6 pour les différentes catégories de détresse psychologique entre les pompiers et les travailleurs québécois (EQCOTESST)

\begin{tabular}{l|c|c}
\hline \multicolumn{1}{c|}{ CATÉGORIE DE DÉTRESSE PSYCHOLOGIQUE } & $\begin{array}{c}\text { Résultats } \\
\text { RQCOTESST } \\
\text { pompiers } \\
\text { (travailleurs } \\
\text { québécois) }\end{array}$ \\
\hline Pas de détresse psychologique (score de 0) & $6 \%$ & $12 \%$ \\
Détresse psychologique faible (score entre 1 et 4) & $66 \%$ & $54 \%$ \\
Détresse psychologique modérée (score de 5 et 6) & $14 \%$ & $15 \%$ \\
Détresse psychologique élevée (score entre 7 et 12) & $13 \%$ & $16 \%$ \\
Détresse psychologique très élevée (score de 13 et +) & $1 \%$ & $3 \%$ \\
\hline
\end{tabular}

\subsection{Prévalence de l'épuisement professionnel}

L'épuisement professionnel est très faible chez les pompiers (tableau 4). En effet, la prévalence de cette condition est de seulement $2 \%$ chez les pompiers. Autrement dit, seulement $2 \%$ des répondants ont un score supérieur à 50 , alors que la prévalence de l'épuisement professionnel est de $49 \%$ chez les préposés 9-1-1.

Tableau 4 : Comparaison des prévalences pour l'épuisement professionnel entre les pompiers et les préposés 9-1-1

\begin{tabular}{l|c}
\multicolumn{1}{c|}{ POPULATION DE COMPARAISON } & Prévalences \\
\hline Pompiers & $\mathbf{2 \%}$ \\
Préposés 9-1-1 & $49 \%$ \\
\hline
\end{tabular}




\subsection{Prévalence du trouble de stress post- traumatique}

Près des trois quarts $(72 \%, 305$ pompiers $)$ des pompiers affirment avoir vécu un événement traumatisant dans leur carrière. Parmi ces 305 pompiers ayant vécu un événement traumatisant dans leur carrière, $1 \%$ présentent une fréquence élevée de symptômes liés au stress posttraumatique (tableau 5). La sévérité des symptômes, pour sa part, est élevée pour $1 \%$ des répondants.
La mesure générale du trouble de stress post-traumatique peut être calculée en combinant les répondants ayant une fréquence élevée et une sévérité élevée: une prévalence de $1 \%$ est alors obtenue. La prévalence est aussi calculée en considérant les répondants ayant obtenu un score total de plus de 50. Avec ce type de mesure, la prévalence de pompiers présentant les symptômes liés au trouble de stress post-traumatique est aussi de $1 \%$. Ce score représente le tiers du score obtenu par les préposés 9-1-1, qui est de $3 \%$.

Tableau 5 : Comparaison des prévalences de fréquence et sévérité des symptômes liés au TSPT et de la mesure totale du trouble de stress post-traumatique entre les pompiers et les préposés 9-1-1

\begin{tabular}{l|c|c}
\hline \multicolumn{1}{c|}{$\begin{array}{c}\text { TROUBLE DE STRESS } \\
\text { POST-TRAUMATIQUE }\end{array}$} & $\begin{array}{c}\text { Prévalences } \\
\text { pompiers }\end{array}$ & $\begin{array}{c}\text { Prévalences } \\
\text { préposés 9-1-1 }\end{array}$ \\
\hline Fréquence élevée des symptômes & $\mathbf{1 \%}$ & $4 \%$ \\
Sévérité élevée des symptômes & $\mathbf{1 \%}$ & $2 \%$ \\
Mesure générale du stress post-traumatique présent & $\mathbf{1 \%}$ & $3 \%$ \\
\hline
\end{tabular}

\subsection{Impact de divers stresseurs liés au travail de pompier}

Les situations impliquant des enfants est l'élément qui dérange le plus les pompiers dans leur travail $(92 \%)$, suivi de près par les interventions qui pourraient impliquer une connaissance des victimes $(90 \%)$.

Le tableau 6 présente en détail la façon suivant laquelle a été évalué chaque stresseur potentiel par les pompiers. Il indique la proportion de réponses à chacun des items et la moyenne accordée à chaque item à la question : "Pour chacun des énoncés, précisez le degré d'impact négatif que l'élément a sur votre santé psychologique ». La toute dernière colonne permet d'avoir une vue d'ensemble de la note moyenne qui a été attribuée à chaque élément. Les items sont présentés, classés en ordre d'importance, selon l'impact négatif qu'ils représentent pour les pompiers, autrement dit, ce qui les dérange le plus dans leur métier. Les pourcentages présentés dans le paragraphe suivant regroupent les fréquences combinées des réponses «assez », « beaucoup » et « extrêmement ».

Les situations impliquant des enfants est l'élément qui dérange le plus les pompiers dans leur travail (92\%), suivi de près par les interventions qui pourraient impliquer une connaissance des victimes $(90 \%)$. Le fait de ne pas pouvoir intervenir lors d'une situation d'urgence à cause de l'attente de renfort est également un élément qui affecte négativement les répondants $(80 \%)$, tout comme le fait d'avoir à intervenir sur des incidents dans lesquels il pourrait y avoir des produits toxiques ou radioactifs (75 \%), ou encore impliquant un risque de contamination biologique (74\%). Parmi les éléments qui ont un impact négatif sur le moins grand nombre de répondants, c'est-àdire les éléments qui dérangent le moins les pompiers, il y a le fait de devoir travailler dans un cadre hiérarchique $(26 \%)$, les foules sur les lieux d'intervention $(17 \%)$, le fait de passer beaucoup de temps en caserne avec les collègues $(11 \%)$ et l'horaire de travail (jour, nuit) $(8 \%)$. 
Tableau 6 : Impact des divers stresseurs liés au travail des pompiers

\begin{tabular}{|c|c|c|c|c|c|c|}
\hline & \multicolumn{5}{|c|}{ Choix de réponses } & \multirow[b]{2}{*}{$\begin{array}{c}\text { Moyenne } \\
\text { /5 }\end{array}$} \\
\hline ITEMS & $\begin{array}{l}\text { Très peu ou } \\
\text { pas du tout } \\
\text { (1) }\end{array}$ & $\begin{array}{c}P e u \\
(2)\end{array}$ & $\begin{array}{c}\text { Assez } \\
\text { (3) }\end{array}$ & $\begin{array}{l}\text { Beaucoup } \\
\text { (4) }\end{array}$ & $\begin{array}{l}\text { Extrê- } \\
\text { mement } \\
\quad(5)\end{array}$ & \\
\hline Situation impliquant un enfant & $2 \%$ & $6 \%$ & $21 \%$ & $37 \%$ & $34 \%$ & $4 / 5$ \\
\hline Connaissance des victimes & $2 \%$ & $8 \%$ & $17 \%$ & $38 \%$ & $35 \%$ & $4 / 5$ \\
\hline $\begin{array}{l}\text { Ne pas pouvoir intervenir en situation } \\
\text { d'urgence à cause de l'attente de renforts }\end{array}$ & $4 \%$ & $16 \%$ & $28 \%$ & $32 \%$ & $20 \%$ & $3.5 / 5$ \\
\hline $\begin{array}{l}\text { Incident avec produits toxiques, } \\
\text { radioactifs }\end{array}$ & $6 \%$ & $19 \%$ & $30 \%$ & $26 \%$ & $19 \%$ & $3.3 / 5$ \\
\hline Risque de contamination biologique & $7 \%$ & $19 \%$ & $30 \%$ & $26 \%$ & $18 \%$ & $3.3 / 5$ \\
\hline $\begin{array}{l}\text { Blessures physiques (de vous-même ou } \\
\text { d'un collègue) }\end{array}$ & $4 \%$ & $24 \%$ & $39 \%$ & $25 \%$ & $9 \%$ & $3.1 / 5$ \\
\hline Panique d'un collègue & $3 \%$ & $23 \%$ & $44 \%$ & $23 \%$ & $8 \%$ & $3,1 / 5$ \\
\hline Manque de matériel & $7 \%$ & $27 \%$ & $33 \%$ & $23 \%$ & $11 \%$ & $3 / 5$ \\
\hline Formation technique estimée insuffisante & $6 \%$ & $28 \%$ & $37 \%$ & $23 \%$ & $7 \%$ & $3 / 5$ \\
\hline Formation théorique estimée insuffisante & $7 \%$ & $33 \%$ & $34 \%$ & $20 \%$ & $7 \%$ & $2.9 / 5$ \\
\hline Détresse, souffrance, décès des victimes & $6 \%$ & $34 \%$ & $35 \%$ & $19 \%$ & $6 \%$ & $2,8 / 5$ \\
\hline $\begin{array}{l}\text { Désaccord entre l'information donnée par } \\
\text { le poste de commandement et la réalité de } \\
\text { l'intervention }\end{array}$ & $8 \%$ & $37 \%$ & $33 \%$ & $17 \%$ & $6 \%$ & $2,8 / 5$ \\
\hline $\begin{array}{l}\text { Responsabilités envers les collègues et les } \\
\text { personnes à secourir }\end{array}$ & $10 \%$ & $34 \%$ & $36 \%$ & $15 \%$ & $5 \%$ & $2,7 / 5$ \\
\hline $\begin{array}{l}\text { Incertitude au sujet de l'évolution de la } \\
\text { situation et de ses conséquences }\end{array}$ & $8 \%$ & $37 \%$ & $40 \%$ & $13 \%$ & $2 \%$ & $2,7 / 5$ \\
\hline $\begin{array}{l}\text { Absence d'aide et d'écoute après un } \\
\text { événement marquant }\end{array}$ & $14 \%$ & $39 \%$ & $26 \%$ & $17 \%$ & $4 \%$ & $2,6 / 5$ \\
\hline Conditions climatiques (chaud, froid) & $14 \%$ & $40 \%$ & $29 \%$ & $14 \%$ & $4 \%$ & $2,5 / 5$ \\
\hline $\begin{array}{l}\text { Difficulté de communiquer efficacement } \\
\text { dû à l'appareil de respiration autonome, } \\
\text { aux bruits, etc. }\end{array}$ & $12 \%$ & $44 \%$ & $27 \%$ & $13 \%$ & $4 \%$ & $2,5 / 5$ \\
\hline Avoir plusieurs rôles en même temps & $10 \%$ & $48 \%$ & $29 \%$ & $12 \%$ & $2 \%$ & $2,5 / 5$ \\
\hline $\begin{array}{l}\text { Climat au travail : entre collègues ou avec } \\
\text { les supérieurs }\end{array}$ & $22 \%$ & $39 \%$ & $23 \%$ & $12 \%$ & $5 \%$ & $2,4 / 5$ \\
\hline Prise de décision dans des délais rapides & $13 \%$ & $50 \%$ & $24 \%$ & $12 \%$ & $1 \%$ & $2,4 / 5$ \\
\hline Étendue de la catastrophe, de l'incendie & $12 \%$ & $51 \%$ & $28 \%$ & $8 \%$ & $1 \%$ & $2,4 / 5$ \\
\hline Affronter une situation nouvelle & $20 \%$ & $47 \%$ & $27 \%$ & $5 \%$ & $2 \%$ & $2,3 / 5$ \\
\hline $\begin{array}{l}\text { Difficultés liées au vêtement de } \\
\text { protection }\end{array}$ & $28 \%$ & $42 \%$ & $17 \%$ & $11 \%$ & $3 \%$ & $2,2 / 5$ \\
\hline $\begin{array}{l}\text { Période de suractivité alternée avec des } \\
\text { périodes d'attente }\end{array}$ & $21 \%$ & $51 \%$ & $21 \%$ & $6 \%$ & $1 \%$ & $2,2 / 5$ \\
\hline Respect de la hiérarchie & $37 \%$ & $38 \%$ & $19 \%$ & $6 \%$ & $1 \%$ & $2 / 5$ \\
\hline Foule sur les lieux d'intervention & $30 \%$ & $53 \%$ & $13 \%$ & $3 \%$ & $1 \%$ & $1,9 / 5$ \\
\hline $\begin{array}{l}\text { Le fait de passer beaucoup de temps en } \\
\text { caserne avec les collègues }\end{array}$ & $54 \%$ & $36 \%$ & $8 \%$ & $3 \%$ & $0 \%$ & $1,6 / 5$ \\
\hline $\begin{array}{l}\text { Horaire de travail (jour, nuit, fin de } \\
\text { semaine) }\end{array}$ & $55 \%$ & $37 \%$ & $5 \%$ & $2 \%$ & $1 \%$ & $1,6 / 5$ \\
\hline
\end{tabular}




\section{DISCUSSION}

Les pompiers, qui sont des travailleurs

d'urgence, soumis à plusieurs risques dans

le cadre de leur travail, présentent une

prévalence à peine plus élevée et parfois

même plus faible que celles obtenues

auprès des travailleurs canadiens ou

québécois.

Outre les comparaisons de prévalences présentées antérieurement, il est possible de constater que, chez les pompiers, la prévalence de la détresse psychologique est similaire aux résultats présentés dans d'autres recherches effectuées auprès de pompiers ${ }^{\mathrm{a}}$. Citons par exemple deux études réalisées auprès de pompiers australiens $^{23 ; 24}$ : la première révèle une prévalence de $27 \%$ (13\% rapportent des signes de détresse psychologique élevée et $14 \%$ modérée) alors que la deuxième présente une prévalence de 24 $\%$ quant à la détresse psychologique des pompiers australiens. Certains auteurs ${ }^{24}$ affirment cependant que ces résultats peuvent être sousestimés, étant donné que les pompiers présentant une forte détresse psychologique sont probablement retirés du service ou en congé de maladie. Il est possible de présumer que cela représente également un biais dans la présente recherche : le taux de détresse psychologique peut être en partie sous-estimé par le fait que seuls les pompiers présents lors du passage en caserne pour distribuer le questionnaire ont participé à la recherche, ce qui signifie que certains pompiers en retrait pour des raisons de maladie n'ont pas été rencontrés. Cependant, il est nécessaire de souligner que le nombre de pompiers absents pour des raisons de maladies n'était pas très élevé (environ 25 sur 155 absents), puisque les autres pompiers absents étaient à l'extérieur de la caserne pour un motif qui n'était pas lié à la santé : vacances, échange de caserne, en formation, etc.

Par contre, la prévalence liée à la détresse psychologique chez les pompiers participant à notre étude est tout de même comparable aux prévalences obtenues auprès des travailleurs canadiens et québécois. Plus précisément, il est possible de constater que les pompiers, qui sont des travailleurs d'urgence, soumis à plusieurs risques dans le cadre de leur travail, présentent une prévalence à peine plus élevée (tableau 1) et parfois même plus faible (tableau 2) que celles obtenues auprès des travailleurs canadiens ou québécois. Il semble donc que les pompiers élaborent des stratégies collectives de défense ${ }^{25}$ qui leur permettent de tenir face aux risques et de maintenir une santé psychologique relativement bonne, tel que le démontre certains auteurs $^{26}$.

Concernant le trouble de stress post-traumatique (TSPT), très peu de pompiers sont touchés par ce trouble (mesure totale : $1 \%$ ) et ce résultat est beaucoup plus faible que celui de bien des recherches faites à travers le monde portant sur le TSPT des pompiers. Par exemple, en Allemagne, une étude ${ }^{27}$ indique que $18 \%$ des pompiers rapportent des symptômes de TSPT; une autre étude montre une prévalence de $22 \%$ pour les pompiers américains ${ }^{28}$ et de $17 \%$ pour les pompiers torontois (Canada) et finalement, une recherche $^{24}$ effectuée auprès de pompiers australiens révèle une prévalence de $9 \%$ quant au TSPT ${ }^{\mathrm{b}}$.

À ce propos, il serait intéressant de savoir si les diverses recherches montrant une forte proportion de TSPT chez les pompiers sont effectuées auprès d'une population ayant vécu un incident critique majeur, par exemple le décès d'un collègue, d'un enfant, l'implication directe dans une intervention qui s'est mal déroulée, etc. Bien entendu, si le questionnaire mesurant le TSPT est passé aux répondants dans les jours suivants un événement d'envergure, il est possible de croire que les résultats seront plus élevés que si le questionnaire est distribué à une population de pompiers qui n'ont pas vécu d'événement traumatisant récemment. Ces données ne sont pas disponibles dans les publications de ces recherches.

De plus, il est important de souligner le fait que l'outil utilisé dans notre recherche (Modified PTSD Symptoms Scale) est un outil de diagnostic servant à identifier les répondants ayant les symptômes d'un stress post-trauma- 
tique présent. Cela signifie que cet outil permet de diagnostiquer seulement les pompiers qui présentent actuellement un TSPT. Cependant, cet outil ne permet pas d'identifier les pompiers qui sont affectés négativement par une situation, mais sans présenter des symptômes liés au TSPT ou encore ceux ayant déjà présenté des symptômes liés au TSPT par le passé.

En outre, la question filtre qui est posée dans notre questionnaire pour déterminer ceux qui devaient répondre aux questions mesurant le TSPT peut porter à confusion. De fait, demander à un pompier s'il a déjà été confronté à un événement traumatisant peut manquer de précision. Certains pompiers ont d'ailleurs expliqué, lors de la passation du questionnaire, qu'ils sont confrontés à ce genre d'événement qui peuvent paraitre traumatisant pour le public, mais qu'eux sont habitués à ce genre d'événements qu'ils ne qualifient plus de traumatisant (ex. : accident d'auto). En d'autres mots, un bon nombre de pompiers (305) ont répondu par l'affirmative à la question demandant s'ils avaient vécu un événement traumatisant au cours de leur carrière, mais très peu sont marqués par cet événement au point de développer un TSPT. Aux fins de comparaison, mentionnons qu'une étude $^{29}$ réalisée auprès du Service d'aide médicale urgente et du Service mobile d'urgence et de réanimation en France (SAMU et SMUR) révèle que $38 \%$ ont vécu au moins un événement traumatisant au cours de leur activité, comparé aux pompiers de la présente étude pour qui ce pourcentage est de $72 \%$. Pour les travailleurs du SAMU et du SMUR, $18 \%$ d'entre eux ont développé un TSPT, alors que dans la présente recherche, seulement $1 \%$ des $72 \%$ ayant vécu un événement traumatisant ont développé un TSPT. Il est donc possible de comprendre que malgré le fait que les pompiers se considèrent parfois exposés à des événements d'ampleur, seulement une petite proportion d'entre eux en sont affectés psychologiquement.

Dans cet ordre d'idées, il est intéressant de constater que les notes moyennes accordées par les répondants aux divers éléments liés au métier de pompier et pouvant avoir un impact négatif sur leur santé psychologique (tableau 6) ne sont pas toutes élevées. En fait, il y a seulement 9 moyennes supérieures à 3/5, sur 28 éléments. En d'autres mots, les pompiers semblent conscients de la nature de leur travail et les éléments qui pourraient provoquer des réactions négatives ne sont pas identifiés par eux comme étant nécessairement dérangeants. Par conséquent, ceci expliquerait que le TSPT soit si bas. En effet, si tous les éléments du tableau 6 avaient été cotés avec une moyenne de $4,5 / 5$ ou $5 / 5$, cela aurait signifié que ces aspects sont plus dérangeants pour les pompiers et auraient alors engendré probablement plus d'impacts sur leur santé psychologique. Cependant, la situation actuelle est telle que ces éléments semblent perçus comme faisant partie intégrante du métier, et les pompiers s'en accommodent bien.

Les situations impliquant des enfants sont les plus pénibles pour les pompiers ainsi que les interventions effectuées auprès de victimes qui sont des connaissances des pompiers.

Toutefois, bien que la prévalence du TSPT ne soit pas élevée et que les moyennes des notes accordées par les pompiers aux divers éléments de ce tableau soient relativement basses, cela n'écarte pas le fait que certaines situations puissent être plus stressantes que d'autres pour les pompiers. Sur ce point, il est en effet possible de constater que les situations impliquant des enfants sont les plus pénibles pour les pompiers ainsi que les interventions effectuées auprès de victimes qui sont des connaissances des pompiers.

Cette liste d'éléments liés à leur travail montre que les pompiers peuvent être touchés par certains aspects bien précis. Cependant, ils ne développent pas tous pour autant un TSPT, bien que ces événements soient parfois marquants. Il est alors possible de comprendre que les pompiers élaborent des stratégies collectives de défense afin de réguler les affects négatifs engendrés par la confrontation aux risques de leur métier, évitant ainsi d'en subir de trop grands impacts au niveau de leur santé psychologique $^{26}$. 


\section{Les pompiers œuvrant dans des villes plus petites ont un risque plus élevé d'intervenir auprès d'une connaissance, ce qui amène d'autres effets sur la santé psychologique $^{24}$. Ils restent par conséquent tout de même à risque de développer des troubles psychologiques suite à certaines interventions.}

Pour revenir à la réflexion concernant les différences de prévalences du TSPT entre notre recherche et celles présentées précédemment, mentionnons que les pompiers d'un pays ou d'une province n'ont pas tous une expérience uniforme quant à la fréquence des interventions $^{24}$. Par conséquent, les pompiers travaillant dans des services incendie des métropoles (Montréal, Sydney, Toronto, Paris, New York, etc.) sont plus fréquemment appelés sur des interventions que les pompiers de villes moyennes ou en milieu rural.

Certes, les pompiers des deux services incendie qui ont participé à notre recherche sont appelés à faire plusieurs interventions, mais il est important de considérer que le nombre d'appels dans une année est relativement moindre que dans des métropoles. Étant donné que pour les pompiers québécois ayant participé à la recherche les interventions représentent une mince partie du travail d'un pompier (environ $10 \%$ de leur temps de travail), l'exposition à ces différents facteurs n'est peut-être pas assez soutenue pour engendrer un haut taux de TSPT. À ce propos, il est important d'être prudent dans la comparaison avec les résultats des autres recherches, qui ont pour la plupart été réalisées dans des services incendie de métropoles (ex. : Toronto, Sydney, etc.).

Par contre, le fait que les pompiers de la présente étude soient moins fréquemment exposés aux risques inhérents à leur métier que d'autres pompiers œuvrant dans des métropoles n'écarte pas la possibilité qu'une intervention en particulier ait un impact sur eux. Par exemple, les pompiers œuvrant dans des villes plus petites ont un risque plus élevé d'intervenir auprès d'une connaissance, ce qui amène d'autres effets sur la santé psychologique ${ }^{24}$. Ils restent par conséquent tout de même à risque de développer des troubles psychologiques suite à certaines interventions.

Soulignons en terminant que, malgré le fait que la santé mentale d'une grande majorité de pompiers soit bonne, certains résultats sont préoccupants. Par exemple, le fait que nous ayons obtenu une prévalence de $1 \%$ quant à la présence de pompiers ayant des symptômes de stress post-traumatique porte à réflexion. Bien que ce pourcentage soit faible, cela signifie tout de même que $1 \%$ des pompiers présents lors de notre passage avec le questionnaire, soit 6 pompiers, ont des symptômes de stress posttraumatique et sont présents au travail. Il faut savoir que si un pompier se présente au travail avec des symptômes de stress post-traumatique, il devient alors un danger pour lui-même et pour ses collègues. Nous nous questionnons donc sur le fait que ces pompiers étaient présents au travail malgré leurs symptômes. Le même constat peut être fait quant à l'épuisement professionnel : les résultats de notre recherche montrent que $2 \%$ de pompiers (10 répondants) étaient en épuisement professionnel lors de notre passage, ce qui est aussi préoccupant.

Par ailleurs, bien que le taux de détresse psychologique soit comparable à la moyenne observée dans la population générale, les personnes ayant des symptômes graves sont considérées comme ayant besoin de soutien psychologique, puisque la détresse psychologique est un indicateur précoce de problèmes de santé psychologique plus graves pouvant survenir si rien n'est fait pour y remédier ${ }^{30}$.

Il faut savoir que si un pompier se présente au travail avec des symptômes de stress post-traumatique, il devient alors un danger pour lui-même et pour ses collègues. Nous nous questionnons donc sur le fait que ces pompiers étaient présents au travail malgré leurs symptômes. 


\section{CONCLUSION}

Les données obtenues à l'aide d'un questionnaire auto-administré auquel 98,6 \% des pompiers approchés ont répondu, nous permettent de constater que l'état de santé psychologique de ces travailleurs est très bon, compte tenu de la dangerosité et des contraintes présentes dans leur travail.

La présente étude poursuivait principalement l'objectif de décrire la prévalence des problèmes de santé psychologique chez les pompiers permanents québécois. Les données obtenues à l'aide d'un questionnaire auto-administré auquel 98,6\% des pompiers approchés ont répondu, nous permettent de constater que l'état de santé psychologique de ces travailleurs est très bon, compte tenu de la dangerosité et des contraintes présentes dans leur travail. Par contre, cette recherche reflète uniquement la situation des pompiers permanents ayant participés à l'étude. Il serait intéressant de s'interroger sur la réalité des pompiers à temps partiel du Québec, qui travaillent sous un mode d'organisation du travail différent, puisqu'ils sont sur appels et ne partagent pas de long quart de travail ensemble, entre les alarmes. Peut-être n'ont-ils pas accès au même type d'environnement de travail que les pompiers à temps plein, lequel permet à ces derniers de tenir dans le métier en bonne santé psychologique?

\section{BIBLIOGRAPHIE}

${ }^{1}$ Morin, E. (2004). «Promouvoir la santé au travail : donner un sens au travail ». Actes du colloque de l'Ordre des conseillers en ressources humaines et en relations industrielles agréés du Québec. Travail et santé psychologique : Crise actuelle et voies de solutions. Sherbrooke. 1-19.

${ }^{2}$ Stansfeld S. et B. Candy (2006). «Psychosocial Work Environment and Mental Health--a Meta-analytic Review ». Scandinavian Journal of Work, Environnement and Health. (32), 443-62.

${ }^{3}$ Maltais, D., S. Robichaud et A. Simard (2001). Sinistres et intervenants. Chicoutimi : JCL.

${ }^{4}$ Duckworth, D.H. (1991). «Facilitating Recovery from Disaster-work Experience ». British Journal of Guidance and Counselling, vol. 19, $\mathrm{n}^{\mathrm{o}}$ 1, 13-22.
${ }^{5}$ Mitchell, J.T. et A. Dyregrov (1993). «Traumatic Stress in Disaster Workers and Emergency Personel - Prevention and Intervention ». In International Handbook of Traumatic Stress Syndromes, sous la dir. de Wilson, J.P et B. Raphael, 905-914. New-York : Plenum Press.

${ }^{6}$ Organisation internationale du travail (2003). «Des meilleures conditions de travail pour les agents des services d'urgence ». Travail, $\mathrm{n}^{\circ} 46$, mars.

${ }^{7}$ Woodall, J.S. (1998). Ask not why Wounded Fall, but how the Valiant Continue to March: New Theory on WorkRelated Stress Management in the Fire-Service. Thèse de doctorat. California: Fielding Graduate University.

${ }^{8}$ DelBen, K., J. Scotti, C. Yi-Chuen et B. Fortson (2006). «Prevalence of Posttraumatic Stress Disorder Symptoms in Firefighters ». Work and Stress, vol. 20, $\mathrm{n}^{\circ}$ 1, 37-48.

${ }^{9}$ Chang, C., L. Lee, K. Canner, J. Davidson et T. Lai (2008). «Modification Effects of Coping on PostTraumatic Morbidity among Earthquake Rescuers ». Psychiatry research, vol. 158, $\mathrm{n}^{\mathrm{O}}$ 2, 164-171.

${ }^{10}$ Cowman, S.E., J.R. Ferrari et M. Liao-Troth (2004). «Mediating Effects of Social Support on Firefighters' Sense of Community and Perceptions of Care ». Journal of community psychology, vol. 32, $\mathrm{n}^{\mathrm{O}} 2,121-126$.

${ }^{11}$ Marchant, R., J. Guernsey et K. Yoshida (1989). Health Effects of Working at the Site of the Gander Arrow Air Crash, Faculty of Medicine, Dalhousie University.

${ }^{12}$ Tak, S., R. Driscoll, B. Bernard et C. West (2007). «Depressive Symptoms among Firefighters and Related Factors after the Response to Hurricane Katrina ». Journal of Urban Health, vol. 84, $\mathrm{n}^{\circ}$ 2. 153-161.

${ }^{13}$ Kessler, R.C., G. Andrew, L. J. Colpe, E. Hiripi, D. K. Mroczek, S.L. Normand, E. E. Walters et A. M. Zaslavsky (2002). «Short Screening Scales to Monitor Population Prevalences and Trends in Non-specific Psychological Distress ». Psychological Medicine, vol. 32, 959-976.

${ }^{14}$ Vézina, M., E. Cloutier, S. Stock, K. Lippel, É. Fortin, et autres (2011). Enquête québécoise sur des conditions de travail, d'emploi, et de la santé et de sécurité du travail (EQCOTESST), Québec, Institut de recherche Robert Sauvé en santé et sécurité du travail - Institut national de santé publique du Québec et Institut de la statistique du Québec.

${ }^{15}$ Furukawa, A., C. Kessler, T. Slade et G. Andrew (2003). «The Performance of the K6 and K10 Screening Scales for Psychological Distress in the Australian National Survey of Mental Health and Well-Being ». Psychological medicine, vol. 33, no 2, 357-362

${ }^{16}$ Kristensen, T.S. et M. Borritz (1999). The Copenhagen Burnout Inventory (CBI); a new questionnaire for measuring burnout. National Institute for Occupational Health.

${ }^{17}$ Resick, R.A., S.A. Falsetti, H.S. Resnick et D.G. Kilpatrick (1991). The Modified PTSD Symptom ScaleSelf Report. St. Louis, MO/Charleston, SC: University of Missouri/National Crime Victims Research and Treatment Center, Medical University of South Carolina. 
${ }^{18}$ Guay, S., A. Marchand, S. Iucci, et A. Martin (2002). «Validation de la version québécoise de l'échelle modifiée des symptômes de trouble de stress posttraumatique auprès d'un échantillon clinique ». Revue québécoise de psychologie, vol. 23, n 3, 257-269.

${ }^{19}$ Ponnelle, S. et X. Vaxevanoglou (1997). «Le stress au quotidien : les sapeurs-pompiers en intervention». Archives des maladies professionnelles, vol. 59, $\mathrm{n}^{\circ} 3$, 190-199.

${ }^{20}$ Marchand, A., A. Demers et P. Durand (2005). «Do Occupation and Work Conditions Really Matter? A Longitudinal Analysis of Psychological Distress Experiences among Canadian Workers ». Sociology of Health and Illness, (27), 602-627.

${ }^{21}$ Toulouse, G., L. St-Arnaud, R. Bourbonnais, J. Damasse, D. Chicoine et A. Delisle (2006). Étude de la prévalence des troubles musculo-squelettiques et psychologiques, des facteurs physiques et psychosociaux chez les préposés des centres d'urgence 9-1-1. Études et recherches / Rapport R-472, Montréal: IRSST.

22. Vézina, M., R. Bourbonnais, A. Marchand et R. Arcand (2008). Stress au travail et santé mentale chez les adultes québécois. Enquête sur la santé dans les collectivités canadiennes (cycle 1.2). Québec: Institut de la statistique du Québec.

${ }^{23}$ Bryant, R. et A. Harvey (1996). «Posttraumatic Stress Reactions in Volunteer Firefighters ». Journal of traumatic Stress, vol. 9, $\mathrm{n}^{\mathrm{o}}$ 1, 51-62.

${ }^{24}$ Dean, G., K. Grow et J. Shakespear-Finch (2003). «Counting the Cost: Psychological Distress in Career and Auxiliary Firefighters ». The Australasian Journal of Disaster and Trauma Studies, vol. 2003-1.

${ }^{25}$ Dejours, C. (2000). Travail, usure mentale. Paris : Bayard.

${ }^{26}$ Douesnard, J. et St-Arnaud, L. (2011) Le travail des pompiers : un métier au service de l'autre, Travailler, vol. $26, \mathrm{n}^{\mathrm{o}} 2,35-53$.

${ }^{27}$ Wagner, D., M. Heinrichs et U. Eklert (1998). «Prevalence of Symptoms of Posttraumatic Stress Disorder in German Professional Firefighters ». American Journal of Psychiatry, vol. 155, $\mathrm{n}^{\mathrm{o}} 12,1727-1732$.

${ }^{28}$ Corneil, W., R. Beaton, A. Murphy, L. Johnson et K. Pike (1999). «Exposure to Traumatic Incidents and Prevalence of Posttraumatic Stress Symptomatology on Urban Firefighters in Two Countries ». Journal of Occupation Health Psychology, (4), 131-141.

${ }^{29}$ Laurent A., K, Chahraouik et P. Carli (2007). «Les répercussions psychologiques des interventions médicales urgentes sur le personnel SAMU : Étude portant sur 50 intervenants SAMU ». Annales médico-psychologiques, vol. $165, \mathrm{n}^{\circ} 8,570-578$.

${ }^{30}$ Vézina, M., M. Cousineau, D. Mergler, A. Vinet et M. Laurendeau (1992). Pour donner un sens au travail : bilan et orientations du Québec en santé mentale au travail. Boucherville : Gäétan Morin.

\section{Notes}

${ }^{\mathrm{a}}$ Ces recherches ont par contre été réalisées avec le General Health Questionnaire (GHQ), ce qui peut causer un biais d'interprétation, bien que l'outil couvre essentiellement les mêmes thèmes.

${ }^{\mathrm{b}}$ Ces recherches ont par contre été réalisées avec le questionnaire Impact Event Scale (IES) ce qui peut en partie expliquer la différence des résultats obtenus dans la présente recherche, bien que l'outil couvre essentiellement les mêmes thèmes. 
ARTS ET LETTRES • Maîtrise en art • Maîtrise en lettres • Maîtrise en linguistique • Doctorat en lettres • SCIENCES HUMAINES • Programme court en études régionales • Maîtrise en études et interventions régionales • Doctorat en développement régional • Diplôme en sciences infimières - Maîtrise en sciences infirmières • Maîtrise en médecine expérimentale • Maîtrise en travail social• Programme court d'accompagnement des aînés •Programmecourten éthique •Programme court en études religieuses • SCIENCES DE L'ÉDUCATION ET DE PSYCHOLOGIE • Diplôme en intervention éducative $\cdot$ Diplôme en enseignement collégial $•$ Maîtrise en éducation • Doctorat en psychologie profil intervention • Doctorat en éducation • Programme court en intervention éducative • Programme court en administration scolaire • Diplôme en administration scolaire • Diplôme en orthopédagogie • SCIENCES ÉCONOMIQUES ET ADMINISTRATIVES • Programme court en gestion de projet • Diplôme en gestion de projet • Maîtrise en gestion de projet • Maîtrise en gestion des organisations - Maîtrise en administration des affaires (MBA) • Diplôme en sciences comptables • SCIENCES APPLIQUÉES • Maîtrise en ingénierie • Doctorat en ingénierie - Diplôme en sciences de la Terre • Maîtrise en sciences de la Terre • Doctorat en ressources minérales • SCIENCES FONDAMENTALES - Maîtrise en ressources renouvelables • Doctorat en sciences de l'environnement • Doctorat en biologie • Programme court en écoconseil • Diplôme en éco-conseil • Programme court de deuxième cycle en gestion durable du carbone forestier • INFORMATIQUE ET MATHÉMATIQUE • Diplôme en informatique appliquée $\cdot$ Maîtrise en informatique • Doctorat en sciences et technologies de l'information

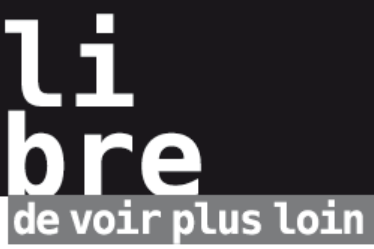

\section{LES ÉTUDES DE CYCLES SUPÉRIEURS}

Consultez nos programmes uqac.ca/programmes

uqac.ca

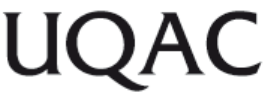

